\title{
Wirtschaftliche Kooperationen innerhalb der neuen EU- Binnengrenzregionen: Voraussetzungen und Entwicklungen entlang der deutsch-polnischen Grenze
}

\author{
Harald Zschiedrich*
}

Mehr als 20 Jahre nach Beginn der Transformationsprozesse und acht Jahre nach der EUOsterweiterung stellt sich die Frage, inwieweit es an den neuen EU-Binnengrenzen bereits zur Herausbildung integrierter Wirtschaftsregionen gekommen ist. Kann man schon von gemeinsamen Wirtschaftsräumen entlang der deutsch-polnischen oder deutsch-tschechischen Grenze sprechen? Welche Rolle spielen in diesem Prozess die Unternehmen? Begegnen sie sich nur als Konkurrenten oder auch als Partner? Sind die Grenzen in einer erweiterten Europäischen Union in wirtschaftlicher, kultureller, administrativer und mentaler Hinsicht schon vollständig überwunden? Oder werden nicht eher mit zunehmender Kooperation und wirtschaftlicher Integration erst recht unterschiedliche Denk-, Sicht- und Handlungsweisen in den Grenzregionen noch spür- und erlebbarer?

Aus der europäischen Geschichte wissen wir, dass mit der Zunahme der europäischen Integration Grenzregionen an Bedeutung gewonnen haben und eine wichtige Rolle beim Zusammenwachsen der europäischen Staaten und Regionen spielen:

„Grenzen trennen, sie verbinden, man kann sich hinter ihnen verstecken, man kann sie überwinden. Grenzregionen werden letztlich daran zu messen sein, ob ihnen dieser Paradigmenwechsel gelingt, diese Blickrichtungsänderung, wo Menschen, die früher Rücken an Rücken standen, plötzlich sich von Angesicht zu Angesicht gegenüber stehen. Die Grenzregionen und ihre Bewohner empfinden sich sehr oft etwas marginalisiert im Vergleich zu den Zentren der Staaten. "

Die nähere Betrachtung zeigt, dass zwischen drei Typen von Grenzregionen zu unterscheiden ist: erstens Grenzgebiete an den staatlichen Grenzen zwischen den alten EU-Mitgliedstaaten; zweitens Grenzregionen zwischen alten und neuen EU-Staaten wie zum Beispiel zwischen Deutschland und Polen oder Tschechien (Regionen wie Ostbayern/Westböhmen, Sachsen/Niederschlesien beziehungsweise die Oder-Neiße-Region); und drittens Grenzregionen an den östlichen EU-Außengrenzen, wie die Grenzgebiete zwischen Polen und Ukraine (beispielsweise die polnische Wojewodschaft Podkarpackie und das Oblast Lwiw in der Ukraine oder die Regionen Białystok und Hrodna in Weißrussland).

Bei allen vorhandenen Unterschieden, Ausgangssituationen und Herangehensweisen zwischen den Regionen Europas besteht die Gemeinsamkeit in der Suche nach neuen, nachhaltigen Wertschöpfungsketten und den meist überall (gleich) geringen finanziellen Spielräumen. ${ }^{2}$

Die wirtschaftliche Bedeutung der Grenzregionen innerhalb der erweiterten Europäischen Union wird allein schon durch die folgenden Fakten erkennbar:

* Prof. Dr. sc. Harald Zschiedrich, Hochschule für Technik und Wirtschaft Berlin.

1 Karl-Heinz Lambertz: Die Euregio: Fakt oder Fiktion?, in: Karl-Heinz Lambertz (Hrsg): Die Grenzregionen als Labor und Motor kontinentaler Entwicklungen in Europa, Zürich/St. Gallen 2010, S. 113-117.

2 Till Meyer: Stärken stärken 2030. Internationalisierung und Europäisierung schaffen das moderne Brandenburg, in: Perspektive 21 51/2011, S. 85-92, hier S. 86-89. 
- Es existieren rund 200 Grenzregionen innerhalb der Europäischen Union.

- Über 30 Prozent der EU-Bevölkerung leben in Grenzregionen, die insgesamt mehr als 40 Prozent des EU-Territoriums einnehmen.

- Die Grenzlinien innerhalb der Europäischen Union sind insgesamt 14.200 Kilometer lang.

- Auch wenn als Grenzregionen statistisch gesehen nur Räume gelten, die maximal 25 Kilometer von der Grenze entfernt sind, so macht diese heute in der ,EU $27^{`}$ einen gesamten Raum von circa 71.000 Quadratkilometern aus, was zweimal der Fläche Deutschlands entspricht.

Der Aufsatz hat folgende Struktur: An die Darstellung der theoretischen Erklärungsansätze für grenzüberschreitende Kooperation schließen sich Betrachtungen zu den Ausgangsbedingungen in den deutsch-polnischen Grenzräumen an. Neben der Bestandsaufnahme und Darlegungen zu aktuellen Entwicklungen werden vor allem Hemmnisse in der Kooperation sichtbar gemacht und Wege zu deren Überwindung aufgezeigt.

Ziel des Aufsatzes ist es, die Ursachen für die noch nicht ausreichende Kooperation zwischen deutschen und polnischen Unternehmen aufzuzeigen und sichtbar zu machen, wo die Besonderheiten im Vergleich zu anderen Grenzregionen liegen.

\section{Theoretische Erklärungsansätze grenzüberschreitender wirtschaftlicher Zusammenarbeit}

Der Autor geht von folgenden drei Thesen aus:

These 1: Mit dem EU-Beitritt Polens und Tschechiens im Jahr 2004 sind die Grenzräume von einer Randlage in das Zentrum der erweiterten Europäischen Union gerückt.

These 2: In dem sich intensivierenden Wettbewerb der Regionen lassen sich Standortvorteile heute nur noch gemeinsam in den Grenzregionen erschließen und vermarkten.

These 3: Die Herausbildung eines grenzüberschreitenden Regionalverständnisses sowie wechselseitigen Vertrauens braucht längere Zeit.

Grenzen haben ihre Rolle in einer erweiterten Europäischen Union noch nicht gänzlich verloren. Ihre Bedeutung hat sich geändert. Bei der Suche nach Erklärungsmustern für grenzüberschreitende wirtschaftliche Kooperationen entsteht somit die grundlegende Frage nach dieser veränderten Bedeutung und Funktion von nationalstaatlichen Grenzen. Da wäre zuerst die klassische und traditionelle Funktion der Abschottung zu nennen, die sich auch und gerade in Zeiten wirtschaftlicher Krisen in dem Streben nach verstärktem nationalem Protektionismus manifestiert. Historisch gesehen hat sich die Bedeutung von Grenzen und damit Grenzregionen stark gewandelt. In den Mittelpunkt drängen heute eher geografische, regionalspezifische, kulturelle und religiöse Aspekte von Grenzräumen, die quasi die heutigen Grenzregionen als ,Kontaktzonen“, ,Brückenköpfe‘ oder ,Drehscheiben“ wichtig machen im Sinne der Vermittlung zwischen unterschiedlichen nationalen Systemen. Michael Daldrup formuliert das wie folgt: „Grenzregionen fungieren heute als Mechanismus zur Lösung von Problemen im europäischen Integrationsprozess und ermöglichen zugleich den Abbau von räumlichen Disparitäten auf europäischer Ebene“. 3

Karl-Heinz Lambertz betont zu Recht, dass Grenzregionen schon etwas ganz besonderes darstellen: „Wo, wenn nicht in Grenzregionen, lässt sich erlebbarer machen, was es heißt Menschen zusammen zu bringen, die aus verschiedenen Staaten kommen, die unterschied-

3 Michael Daldrup: Funktionslogik von grenzüberschreitenden Kooperationen, Berlin 2005, S. 29. 
liche Sprachen sprechen, die unterschiedliche Mentalitäten haben und die auch sonst verschieden sind."4

Die regionalökonomische Forschung weist auf den Wandel hin, der sich schließlich in der Geografie der Märkte vollzieht. Dem liegt die Überlegung zugrunde, wonach sich das Marktpotenzial infolge der Öffnung der mittel- und ost-europäischen Märkte in Richtung Osten verschiebt. Mit der erfolgreichen Transformation in den neuen EU-Mitgliedstaaten Mittelosteuropas, ihrer Einbindung in internationale Produktionssysteme und globale Wertschöpfungsketten infolge gewachsener Zuströme an ausländischen Direktinvestitionen hat sich die obige skizzierte Tendenz (Verschiebung des Marktpotenzials nach Osten) noch weiter verstärkt. Diese Diskussion ist nicht generell neu, denn der Ansatz, wonach sich Marktpotenziale durch wirtschaftliche Integrationsprozesse verschieben, wurde bereits im Zusammenhang mit der Theorie der neuen ökonomischen Geografie von Paul Krugman ${ }^{5}$ entwickelt. Die Regionalökonomie ist zu folgenden Erkenntnissen gelangt: Firmen können aus Grenzräumen ,vertrieben' werden, weil ihnen dort ein Teil des Marktes abgeschnitten wird. Alleinstellungsmerkmale, Spezialisierungen, regionale Verbünde und Cluster gewinnen auch in Grenzregionen an Bedeutung. Das Marktpotenzial hat einen wichtigen Einfluss auf die Standortverteilung. Es entwickeln sich jene Regionen schneller und stärker, die wettbewerbsfähigen Auslandsmärkten näher sind. Ein Beispiel sind ,Maquiladora'-Industries an der US-amerikanisch-mexikanischen Grenze. Maquiladoras sind Produktions- oder Montagebetriebe entlang der mexikanisch-US-amerikanischen Grenze. Das Besondere dieser Betriebe ist, dass Rohstoffe und Komponenten zur Weiterverarbeitung zollfrei importiert werden, um nach dem Bearbeitungsprozess in Mexiko wieder in die USA reexportiert werden.

Die theoretische Diskussion widmet sich auch zunehmend dem Entstehen von grenzüberschreitenden Verflechtungsräumen. Was dabei die deutsch-polnische Grenzregion angeht, so ist es verfrüht, bereits von Verflechtungsräumen zu sprechen. Indikatoren dafür sind intensive Handelsbeziehungen, Ströme von Direktinvestitionen, eine gleich verlaufende Entwicklung auf den Arbeitsmärkten sowie eine Angleichung bei den Löhnen, der Kaufkraft und den Energie- und Bodenpreisen. Im Vordergrund dabei stehen die Indikatoren, „,welche die für Grenzregionen typische Verflechtungen und Integrationsprozesse über nationale Grenzen hinweg abbilden. Von besonderem Interesse sind beispielweise Pendelverflechtungen in den Sektoren Arbeit und Ausbildung, Wohnen und Freizeit oder grenzüberschreitende Unternehmenskooperationen und Wertschöpfungsketten." ${ }^{\text {“6 }}$

Beispiele hierfür finden sich in Löcknitz (Landkreis Uecker-Randow in MecklenburgVorpommern). Dort leben inzwischen 200 polnische Familien. Polnische Kinder besuchen die Löcknitzer Europa-Schule. Positive Trends zeichnen sich auch in Pasewalk ab, wo gemeinsame Unternehmensmessen stattfinden nach dem Motto: „Ohne Grenzen“ beziehungsweise „Bez Granic“.

\section{Ausgangsbedingungen für ,cross-border cooperation' in deutsch-polnischen Grenzregionen}

Betrachten wir die spezifischen Ausgangsbedingungen für den Ausbau der grenzüberschreitenden deutsch-polnischen Unternehmenskooperationen, so zeigen sich im Einzelnen

4 Karl-Heinz Lambertz: Gemeinsames Erinnern europäischer Zeitzeugen, in: Karl-Heinz Lambertz (Hrsg): Die Grenzregionen als Labor und Motor kontinentaler Entwicklungen in Europa, Zürich/St. Gallen 2010, S. 170-180.

5 Vgl. Paul Krugman/Maurice Obstfeld: Internationale Wirtschaft. Theorie und Politik der Außenwirtschaft, München 2010.

6 Andrea Hartz/Gerd-Reiner Damm/Stefan Köhler: Großräumige grenzüberschreitende Verflechtungsräume, in: Raumforschung - Raumordnung 6/2010, S. 499-513, hier S. 510. 
folgende Besonderheiten: Der EU-Binnenmarkt, die EU-Assoziierungsabkommen und die nunmehr achtjährige volle EU-Mitgliedschaft Polens haben bisher noch keine neuen, weiteren und zusätzlichen Anreize für die grenzüberschreitende Kooperation im Güter- und Dienstleistungssektor geschaffen. Neue industrielle Strukturen als Ergebnis grenzüberschreitender struktur-politischer Überlegungen sind nicht vorhanden. Bestehende Industriekerne (zum Beispiel der Schiffbau in Stettin) sind im Transformationsprozess auf der polnischen Seite stark geschrumpft. Aber auch die im Zuge der deutschen Einheit stattgefundene Deindustrialisierung in Grenzregionen der neuen Bundesländer hat keinen Nährboden geschaffen, auf dem regionale Kerne oder Cluster entstehen konnten. Auf beiden Seiten der Grenze sind die kleinen und mittleren Unternehmen (KMU) in Industrie und Handwerk zu wenig exportorientiert und international nicht ausreichend vernetzt. Dabei macht die Ausrichtung des Handwerks vorwiegend auf den regionalen Bedarf diesen Sektor eigentlich sehr geeignet für die grenzüberschreitende Zusammenarbeit. Die Gründung eigener Niederlassungen entlang der Grenze ist dann sinnvoll, wenn:

1. die handwerkliche Leistung eine unmittelbare Marktnähe/-präsenz notwendig macht (zum Beispiel Optiker, Bäckereien, KFZ-Reparaturhandwerk, Gesundheits- und Ernährungshandwerk);

2. im anderen Staat des Grenzraumes ein langfristiges Engagement geplant ist;

3. ein Unternehmen damit ein zweites Standbein errichten will;

4. der Einsatz polnischen beziehungsweise tschechischen Personals gewünscht ist oder nötig wird aus Arbeitskostengründen oder auch aus Kapazitätsüberlegungen (möglich ist auch die Nachfrage nach deutschen Arbeitskräften zum Beispiel in polnischen Clusterregionen, wo nach dem Abwandern von circa 2 Millionen jungen polnischen Arbeitskräften nach Irland, England, Schweden etc. ein akuter Fachkräftemangel herrscht; wie in Wroclaw, Katowice, Poznan, Warschau etc.);

5. aus langfristiger Sicht betriebswirtschaftliche Kostenvorteile eine Niederlassung sinnvoll machen. ${ }^{7}$

Innovationspotenziale und damit auch die Chancen für neue exportorientierte Produktionen sind wenig entwickelt. Die Investitionen in Forschung und Entwicklung erreichten im Jahr 2005 mit einem Anteil von 1,2 Prozent am Bruttoinlandsprodukt nur etwa die Hälfte des ostdeutschen beziehungsweise gesamtdeutschen Wertes (2,4 Prozent beziehungsweise 2,5 Prozent!). Je 1.000 Erwerbstätige sind in Mecklenburg-Vorpommern nur 1,4 Personen in Forschung und Entwicklung beschäftigt. In Ostdeutschland sind es im Durchschnitt 3,3 und in Gesamtdeutschland 8,3. Der bestehende Forschungs- und Entwicklungssektor in der Grenzregion Mecklenburg-Vorpommerns wird wesentlich durch die öffentliche Hand getragen. Das industrielle Verwertungspotenzial von Forschungs- und Entwicklungsresultaten fehlt weitgehend in der Region, was die Unternehmen eher zur überregionalen Kooperation veranlasst. Der Internationalisierungsgrad der polnischen KMU ist noch wenig vorangeschritten. Die meisten polnischen Firmen in den Grenzregionen konzentrieren sich auf die lokalen Märkte und haben noch eine geringe Innovationskraft.

Resümiert man die Besonderheiten in den Ausgangsbedingungen für die Kooperation an der deutsch-polnischen Grenze, so fallen zwei Punkte ins Gewicht:

Erstens im Unterschied zur deutsch-polnischen Grenze, existiert in den Wirtschaftsräumen entlang der deutsch-tschechischen Grenze (Sachsen/Nordböhmen und Bayern/West-

7 Vgl. Harald Zschiedrich: Grenzregionen als Wegbereiter von Internationalisierung und wirtschaftlicher Integration - Erfahrungen aus der deutsch-polnischen Kooperation, in: Harald Zschiedrich (Hrsg.): Wirtschaftliche Zusammenarbeit in Grenzregionen, Berlin 2011, S. 13-40, hier S. 32. 
böhmen) ein verarbeitendes Gewerbe mit hoher Exportintensität. In diesen Regionen hat die EU-Erweiterung in der Tat zu einem Schub in der Kooperation zwischen den Unternehmen geführt.

Zweitens Innovative Wirtschaftssektoren sind bis auf wenige Ausnahmen (Frankfurt Oder und Stettin) an der deutsch-polnischen Grenze kaum zu finden. Ausländische Investoren sind wenig präsent. Ausnahmen bilden Frankfurt Oder und Eisenhüttenstadt. Dagegen profitieren in Süddeutschland die grenzüberschreitenden Kooperationen von der bayerischen Clusterinitiative.

\section{Schwache Exportintensität in den Grenzregionen}

Nur wenige leistungsstarke Unternehmen sind in die regionale und internationale Arbeitsteilung eingebunden, wobei die Bandbreite des Sortiments der Produkte zum Beispiel in Brandenburg mit Hafenkränen, Werkzeugmaschinen, montagebereiten Baugruppen, Komponenten für Windkraftanlagen etc. durchaus attraktiv ist. Insgesamt werden die Möglichkeiten in den Grenzregionen für die Vertiefung des deutsch-polnischen Außenhandels bei Weitem noch nicht voll ausgeschöpft, wovon auch die nachfolgenden Fakten zeugen:

Die vier für die polnischen Außenhandelsbeziehungen wichtigsten deutschen Bundesländer sind schon seit geraumer Zeit Nordrhein-Westfalen, Bayern, Niedersachsen und BadenWürttemberg. Auf diese vier entfällt ein Gesamtanteil von weit mehr als 60 Prozent am deutsch-polnischen Außenhandel. Allerdings kann auch festgehalten werden, dass für die neuen Bundesländer, die über eine gemeinsame Grenze mit Polen verfügen, die Handelsbeziehungen mit Polen prozentual bedeutsamer sind als für die deutsche Wirtschaft insgesamt. Schließlich war Polen unter den Schwellenländern ${ }^{8}$ im Jahr 2009 für Deutschland der zweitwichtigste Exportmarkt hinter China; bis zum Jahr 2008 sogar der wichtigste Exportmarkt. In der gesamten deutschen Exportstatistik liegt Polen auf dem zehnten Platz und beim Import an zwölfter Stelle. Wie sieht es nun mit dem Stellenwert der deutsch-polnischen Handelsbeziehungen in den Grenzregionen (Brandenburg, Sachsen) aus?

Tabelle 1 zeigt den Gesamtüberblick über die Anteile der einzelnen Bundesländer am deutsch-polnischen Warenaustausch aus polnischer Sicht.

Die Tabelle 1 macht deutlich, dass eben nicht nur die räumliche Nähe zu Polen entscheidend für die Stellung der einzelnen Regionen im Außenhandel ist. Offenbar spielt das Vorhandensein wettbewerbsfähiger Großunternehmen sowie exportorientierter KMU und zahlreicher ,hidden champions', leistungsstarker Infrastrukturen, gut funktionierender Zulieferernetzwerke und vorhandener Industrie- und Dienstleistungscluster in den ,Spitzenreitern unter den Bundesländern, Bayern, Baden-Württemberg, Nordrhein-Westfalen und Niedersachsen, eine ganz entscheidende Rolle. Die Unterschiedlichkeit in der Ausstattung mit Standortfaktoren für Kooperationen und Investitionen betreffen vor allem die Zukunftsbranchen, die Bevölkerungsdichte, die Einkommensunterschiede zwischen den Agglomerationsräumen und den ländlichen Regionen beiderseits der deutsch-polnischen Grenze. Bei der Interpretation von Tabelle 1 ist zu beachten, dass die in den neuen Bundesländern agierenden Unternehmen häufig als Zulieferer für exportorientierte Großunternehmen in den alten Bundesländern operieren und somit ihre Finalprodukte nicht in die Exportstatistik eingehen. Des Weiteren ist zu berücksichtigen, dass heute vielfach Direktinvestitionen den Boden für Handelsströme bereiten. Allerdings sind die Regionen entlang der deutsch-polnischen Grenze

8 Gruppe von 26 ,emerging markets'. 
Tabelle 1: Deutsch-polnischer Außenhandel 2009 (nach Bundesländern - Anteil am Gesamtumsatz)

\begin{tabular}{|l|c|}
\hline \multicolumn{1}{|c|}{ Bundesland } & $\begin{array}{c}\text { Anteil in } \\
\text { Prozent }\end{array}$ \\
\hline Nordrhein-Westfalen & 24,5 \\
\hline Bayern & 13,1 \\
\hline Niedersachsen & 12,0 \\
\hline Baden-Württemberg & 11,4 \\
\hline Hessen & 6,6 \\
\hline Brandenburg & 5,4 \\
\hline Sachsen & 4,7 \\
\hline Hamburg & 3,9 \\
\hline Sachsen-Anhalt & 3,8 \\
\hline Rheinland-Pfalz & 3,7 \\
\hline Berlin & 2,9 \\
\hline Schleswig-Holstein & 2,1 \\
\hline Thüringen & 1,8 \\
\hline Mecklenburg-Vorpommern & 1,5 \\
\hline Bremen & 1,4 \\
\hline Saarland & 1,3 \\
\hline
\end{tabular}

Abweichungen sind rundungsbedingt. Quelle: Jacek Robak: Stand und Perspektiven des deutsch-polnischen Außenhandels, in: Vortrag auf der Jahrespressekonferenz der polnischen Handelsvertretung, Berlin 2010.

bisher keine bevorzugten Empfänger von ausländischen Direktinvestitionen. So gehen nur 3 Prozent aller polnischen Auslandsinvestitionen nach Deutschland in Grenzregionen. Dennoch kann festgestellt werden, dass vor allem in Sachsen und Brandenburg die wirtschaftlichen Verflechtungen mit Polen wachsen: „Das Ausfuhrvolumen von Sachsen und Brandenburg nach Polen betrug im Jahr 1992 rund 98,3 Mill. Euro und in die Tschechische Republik 132,1 Mill. Euro. Die Nachfrage nach sächsischen Gütern stieg bis 2010 kräftig an, sodass der Freistaat Sachsen Güter im Wert von 1,4 Mrd. Euro nach Polen und 1,2 Mrd. Euro nach Tschechien exportierte."9 Eine Studie des Hamburger Weltwirtschaftsarchives (HWWA) liefert weitere interessante Daten: Danach betrug der Anteil Polens an den Exporten Brandenburgs 12,6 Prozent sowie Sachsen-Anhalts 12,7 Prozent (2011). Dagegen beträgt der Anteil Polens an den Exporten Hamburgs 2,9 Prozent wobei Hamburg 1,9 Prozent seiner Importe aus Polen bezieht und 1,3 Prozent aus Tschechien. Insgesamt macht diese

9 Robert Lehmann/Johannes Steinbrecher: Wirtschaftliche Verflechtungen Sachsens mit seinen Nachbarregionen in Polen und Tschechien, in: ifo Dresden 2/2012, S. 3-12, hier S. 6. 
Studie sichtbar, dass die Bedeutung der deutschen Absatzmärkte für die tschechischen Exporteure stärker ausgeprägt ist als für die polnischen Unternehmen. ${ }^{10}$

Untersuchungen haben in diesem Zusammenhang ergeben, dass die Leistungstiefe, die Leistungsfähigkeit und das Niveau der Produktivität in strukturschwachen Grenzregionen die mögliche Einbindung in die internationale/regionale Arbeitsteilung stark beeinflussen können: „Während die leistungsstarken Wirtschaftseinheiten intensiv in die regionale Arbeitsteilung (innerhalb der Region Berlin/Brandenburg) eingebunden waren, operierten andere Unternehmen mit einer relativ hohen Leistungstiefe (hohe Eigenfertigung ohne Kooperationen) oder bezogen die erforderlichen Vorleistungen mit hohem Aufwand aus weiter entfernten Gebieten." "11

Daraus folgt, dass aus regional lückenhaften Zuliefer- und Abnehmernetzwerken sich mitunter größere Entfernungen zu den geeigneten Kooperationspartnern ergeben. Sofern also Unternehmen in strukturschwachen (Grenz-)Regionen beabsichtigen, den Umfang ihrer Eigenleistung konsequent wirtschaftlich zu gestalten, sehen sie sich in vielen Fällen veranlasst, die erforderlichen Zukäufe aus weiter entfernten Gebieten (Baden-Württemberg, NordrheinWestfalen unter anderem) zu beziehen. Daraus ergeben sich wiederum längere Lieferzeiten sowie höhere Beschaffungskosten, was die internationale Wettbewerbsfähigkeit der KMU negativ beeinflusst.

Jörn Mallok schlägt mit Blick auf künftige Ansiedlungen und die Wirtschaftsförderung in den Grenzregionen Brandenburgs vor: „Sofern sich offene Positionen im Unternehmensbestand des untersuchten Industriekernes durch passgenaue Ansiedlungen besetzen lassen, könnten nachfragende Unternehmen den aufwendigen Bezug von überregionalen Kooperationsleistungen durch kostengünstige sowie zeitnahe Lieferungen aus dem eigenen Gebiet ablösen." 12

\section{Bestandsaufnahme der Kooperation in deutsch-polnischen Grenzregionen}

Bekanntlich ist es der polnischen Wirtschaft in den vergangenen Jahren recht gut gelungen, zunehmend ausländische Investoren ins Land zu holen. Dabei ist allerdings auch zu konstatieren, dass sich unter den ersten zehn Subregionen nur eine Grenzregion an der deutsch-polnischen Grenze befindet. ${ }^{13}$

Positiv ist die Entwicklung im Großraum Stettin zu bewerten, denn bei den letzten Rankings lag dieser noch auf dem 13. Platz der Analyse des Danziger Institutes für Marktforschung. In der Tabelle 2 sind auch Subregionen aufgeführt die keine Grenzregionen sind.

Die Verbesserung ist zurückzuführen auf die Bemühungen um eine leistungsstärkere Infrastruktur sowie das Arbeitsplatzangebot. Schließlich ging in der Subregion Stettin auch der Anteil der dort ausgewiesenen Schutzgebiete zurück, was neue Chancen für industrielle Entwicklung in der Grenzregion eröffnet. Für den Ausbau der grenzüberschreitenden Kooperation im Raum Stettin sprechen ferner die gute Transportzugänglichkeit/gute Verkehrsanbindungen zur Westgrenze Polens und eine wachsende Anzahl von Firmen mit der Beteiligung von ausländischem Kapital. Als Hemmnisse in der Grenzregion Stettin erweisen

10 Vgl. Michael Bräuniger/Silva Stiller/Mark-Oliver Teuber/Jan Wedermeier: Ökonomische Entwicklungsperspektiven in der Kammerunion Elbe/Oder (KEO), in: HWWI Policy Report Nr. 18, 2012, S. 10-70.

11 Jörn Mallok: Leistungstiefe und Leistungsfähigkeit metallverarbeitender Unternehmen in strukturschwachen Regionen, in: Deutsche Betriebswirtschaft 3/2008, S. 323-336.

12 Ebenda, S. 333.

13 Der Wirtschaftsraum Polen ist in 16 Wojewodschaften und 66 Subregionen eingeteilt. 
Tabelle 2: Investitionsattraktivität der Subregionen für die Industrie: Rangliste nach Plätzen 2009-2010

\begin{tabular}{|l|c|c|}
\hline \multicolumn{1}{|c|}{ Subregion } & $\mathbf{2 0 0 9}$ & $\mathbf{2 0 1 0}$ \\
\hline Kattowitz & 1 & 1 \\
\hline Rybnik & 2 & 2 \\
\hline Lodz & 3 & 4 \\
\hline Breslau & 7 & 5 \\
\hline Bielsko-Biała & 11 & 6 \\
\hline Posen & 4 & 7 \\
\hline Bromberg-Thorn & 5 & 8 \\
\hline Auschwitz & 9 & 9 \\
\hline Krakau & 8 & 10 \\
\hline Stettin & 13 & 3 \\
\hline
\end{tabular}

Quelle: Die Investitionsattraktivität der Wojewodschaften und Subregionen Polens, Danziger Institut für Marktwirtschaft, Gdansk 2010.

sich - laut Danziger Institut für Marktforschung - die relativ hohen Arbeitskosten im Vergleich zu anderen polnischen Subregionen. Auch die sehr hohe Kriminalität in der Subregion Stettin erweist sich laut Danziger Studie als ein Hemmnis für die ausländischen Investoren. ${ }^{14}$

Hoffnungsvoll stimmt die stetige Zunahme von KMU in der gesamten Wojewodschaft Zachodniopomorskie (Westpommern), in der Stettin liegt. Auch bilden stark entwickelnde Industrieparks in Goleniow, Police, Karlin, Koszalin sowie Gryfin und Walcz eine gute Basis für künftige grenzüberschreitende Kooperationen. Neben dem Tourismus bieten sich deutschen KMU vor allem Möglichkeiten in den Branchen Elektromaschinenbau, Chemie und in der Holz und Lebensmittel verarbeitenden Industrie.

Einzelne positive Entwicklungen können jedoch insgesamt nicht darüber hinwegtäuschen, dass neu entstehende Projekte, Strukturen auf beiden Seiten der deutsch-polnischen Grenze sich noch zu wenig ergänzen, um eine effiziente Basis für den angestrebten gemeinsamen Wirtschaftsraum entlang der Grenze zu entwickeln im Gegensatz zu grenzüberschreitenden Verflechtungsräumen in einigen alten Bundesländern wie zum Beispiel am Bodensee, am Oberrhein und in der Region Aachen. Aber auch im Wirtschaftsraum Oberpfalz/Kehlheim und Westböhmen sind die Bemühungen um grenzüberschreitende unternehmerische Netzwerke im verarbeitenden Gewerbe wesentlich weiter vorangekommen als im deutsch-polnischen Grenzraum. ${ }^{15}$ Indikatoren hierfür sind eine höhere Exportorientierung der Unternehmen zum Beispiel im Kreis Pilsen sowie eine stärkere Internationalisierung des Dienstleistungssektors in Westböhmen bei deutlicher Verringerung des Einkommensabstandes

14 Vgl. Anna Hildebrandt/Marcin Nowicki/Przemyslaw Susmarski/Maciej Tarkowski: Die Investitionsattraktivität der Wojewodschaften und Subregionen Polens, Danziger Institut für Marktwirtschaft, 2010, S. 5-67.

15 Vgl. Jürgen Jerger/Michael Knogler: Regionale Aspekte wirtschaftlicher Integration: Das Fallbeispiel der grenzüberschreitenden Zusammenarbeit zwischen Oberpfalz/Westböhmen, in: Harald Zschiedrich (Hrsg.): Wirtschaftliche Zusammenarbeit in Grenzregionen, Berlin 2011, S. 65-82. 
zwischen Westböhmen und Ostbayern. Es wird in dieser Region deutlich, dass auf beiden Seiten der deutsch-tschechischen Grenze die Unternehmen die regionale Kooperation immer mehr zu einem Standortfaktor in der harten Konkurrenz um neue Märkte und Clusterpotenziale machen.

Große Reserven im deutsch-polnischen Wirtschaftsraum liegen also in einer engeren Verzahnung, Koordinierung der Projekte und aktuellen Planungen, was auch am Beispiel der ,twin-city‘ Frankfurt Oder/Slubice deutlich wird (siehe dazu Tabelle 3).

Tabelle 3: Gegenüberstellung von Merkmalen wirtschaftlicher Entwicklung zwischen den beiden Grenzstädten Frankfurt Oder und Slubice

\begin{tabular}{|l|l|l|}
\hline Vergleichskategorie & \multicolumn{1}{|c|}{ Frankfurt Oder } & \multicolumn{1}{c|}{ Slubice } \\
\hline Einwohnerzahl & circa 60.000 & circa 17.000 \\
\hline Branchenschwerpunkte & $\begin{array}{l}\text { Mikroelektronik, Solartech- } \\
\text { nik, Umwelttechnologie, } \\
\text { Kommunikationstechnik }\end{array}$ & $\begin{array}{l}\text { Holzverarbeitung, Möbelin- } \\
\text { dustrie, Metallverarbeitung, } \\
\text { Nahrungsmittelproduktion }\end{array}$ \\
\hline $\begin{array}{l}\text { Potenziale für neue An- } \\
\text { siedlungen }\end{array}$ & $\begin{array}{l}\text { Großflächige Industrie- } \\
\text { ansiedlungen }\end{array}$ & $\begin{array}{l}\text { Gute Entwicklung von Ge- } \\
\text { werbegebieten und Einrich- } \\
\text { tung von Sonderwirtschafts- } \\
\text { zonen }\end{array}$ \\
\hline Wirtschaftsförderung & $\begin{array}{l}\text { Zahlreiche Einrichtungen } \\
\text { sind vorhanden }\end{array}$ & $\begin{array}{l}\text { Umfangreiche Fördermög- } \\
\text { lichkeiten existieren }\end{array}$ \\
\hline Infrastruktur & $\begin{array}{l}\text { Ausbau der Eisenbahn-Stre- } \\
\text { cke Frankfurt/Oder-Poznan- } \\
\text { Warsaw }\end{array}$ & $\begin{array}{l}\text { Erneuerung der Oder-Brücke, } \\
\text { verbesserte Ortsumfahrung }\end{array}$ \\
\hline
\end{tabular}

Quelle: Eigene Darstellung.

Ein Vergleich dieser Charakteristika der beiden Teile der Doppelstadt Frankfurt Oder/ Slubice macht deutlich, dass die Branchenstrukturen voreinander abweichen. Daraus folgt, dass zwangsläufig eine relativ geringe Kooperationsneigung zu erwarten ist. Künftig wird es deshalb darum gehen, das Denken und Handeln der Verantwortlichen für die grenzüberschreitende Kooperation von Beginn an mehr auf die Erfordernisse des Gesamtraumes zu richten, nicht nur um Fehlplanungen zu vermeiden, sondern vielmehr die Chancen abgestimmter arbeitsteiliger Entwicklungen bewusster wahrzunehmen. Somit muss in den Grenzregionen Mecklenburg-Vorpommern, Brandenburg und Sachsen noch stärker auf die europäische Ausrichtung fokussiert werden, was eine internationale Öffnung von entstehenden regionalen Clustern einschließt. ${ }^{16}$

Resümierend ergibt eine Bestandsaufnahme folgendes Bild: Die Unternehmenskooperationen im Grenzraum erfassen bislang nur wenige Branchen, zum Beispiel den Bausektor, die Metallverarbeitung, den Holzsektor, die Bekleidungsindustrie und den Handel. Deutsche Direktinvestitionen in Polen konzentrieren sich nur auf einige Zweige, insbesondere den Chemie-, Möbel- und Lebensmittelsektor. Es gibt zu wenige wettbewerbsfähige Zulieferunternehmen, die sich gut einpassen in die betriebliche Arbeitsteilung der in den Grenzregionen ansässigen deutschen und polnischen KMU. Die Unternehmen, die keine geeigneten

16 Vgl. Meyer: Stärken stärken 2030, 2011, S. 85-92. 
Kooperationspartner im Vertrieb, in der Fertigung, im Einkauf oder in der Entwicklung finden, weichen in der Regel auf überregionale Kooperationspartner aus und erhöhen somit ihre eigene Fertigungstiefe. ${ }^{17}$ Vielen KMU haben Informationsdefizite über potenzielle Märkte, Kontaktanbahnungen und Fördermöglichkeiten im jeweils anderen Staat. Die personellen Kapazitäten sind gering und die Mitarbeiter verfügen nicht immer über ausreichende interkulturelle Kompetenz. Es bestehen größenbedingte Nachteile von KMU, so zum Beispiel bei der Auftragsabwicklung oder bei der Kontaktpflege sowie der Vereinbarung von Einkaufspreisen. Die KMU in den Grenzregionen haben geringe Exportquoten: Brandenburg 26 Prozent und Sachsen 23 Prozent, im Vergleich dazu Deutschland gesamt 42 Prozent.

\section{Kooperationshemmnisse}

Neben den allgemeinen Barrieren wie fehlende Kenntnisse in der Sprache des Kooperationspartners, bestehen entlang der deutsch-polnischen Grenze eine Reihe von spezifischen Hemmnissen:

Erstens reichen die fachlichen Kenntnisse vieler Unternehmer auf dem Gebiet der Außenwirtschaft noch nicht aus.

Zweitens gibt es nicht nur mangelndes Interesse der Unternehmer, sondern auch starke Hemmungen, in der Region grenzüberschreitend aktiv zu werden.

Drittens treffen in den Grenzräumen föderal geprägte wirtschaftlichen Strukturen in Deutschland auf eher zentralistisch geprägte Denk- und Handlungsmuster in Polen.

Viertens gibt es zu wenige Großunternehmen, die auf internationalen Märkten produzieren und investieren.

Fünftens geht die Zahl der Unternehmen in den Grenzregionen zurück, was einhergeht mit einer zunehmenden Abwanderung der jungen Bevölkerung.

Sechstens ist die Ausbreitung der Innovationen in den Grenzregionen zu langsam.

Siebtens zielen die Entwicklungskonzepte sehr einseitig auf endogene Ressourcen innerhalb der Grenzregionen.

Achtens kam die Öffnung des Arbeitsmarktes zu spät (1. Mai 2011), bedingt durch die Festlegung von Übergangsfristen für die Arbeitnehmerfreizügigkeit. Ursache dafür waren die ursprünglich gemachten Schätzungen in Österreich und Deutschland, die von einem sehr hohen Migrationspotenzial ausgegangen sind (160.000-380.000 pro Jahr in Deutschland). Die zu langen Übergangsfristen waren nicht förderlich für die grenzüberschreitende Kooperation, insbesondere im Dienstleistungssektor. Im Jahr 2009 waren in Brandenburg nur knapp 1.800 polnische Arbeitnehmer sozialversicherungspflichtig beschäftigt. Das waren lediglich 1,9 Prozent aller in Deutschland sozialversicherungspflichtig beschäftigten polnischen Arbeitskräfte. Dagegen sind in Nordrhein-Westfalen 24 Prozent beschäftigt. ${ }^{18}$

Um diese Hemmnisse abzubauen, bedarf es künftig mehr gemeinsamer Planungen und Absprachen. Es müssen mehr KMU stimuliert werden, grenzüberschreitend tätig zu werden. Auch sollte die Vermarktung von Grenzregionen nach außen gemeinsam in Angriff genommen werden. Ein Beispiel ist die Regionalmarke Uckermark.

17 Überregionale Kooperationen werden immer dann erforderlich, wenn Unternehmen in ihrer eigenen Region keinen geeigneten Partner finden. Das kann auch dazu führen, dass mehr Fertigung in eigener Regie durchgeführt wird.

18 Vgl. Wolfgang Schröder: Das Ende der Übergangsfristen: Soziale und politische Auswirkungen in den Grenzregionen, in: Eva Kocher/Cartsen Nowack (Hrsg): Freie Fahrt für Arbeitnehmer/innen zwischen Ost und West, Baden-Baden 2012, S. 69-77. 
Die wirtschaftsfördernden Maßnahmen müssen viel stärker als bisher an die konkreten Interessen und Probleme der Unternehmen angepasst werden, wenn eine Wirkungslosigkeit von Fördermaßnahmen in den Grenzregionen vermieden werden soll. Allerdings kann die beste Wirtschaftsförderung nicht die Eigeninitiative der Unternehmer in den deutsch-polnischen Grenzregionen ersetzen.

Die wichtigste ,Ressource‘ für die Überwindung der skizzierten Barrieren ist die Schaffung gegenseitigen Vertrauens, der Abbau einer „Kultur des Misstrauens“. Das beginnt beim fehlenden Vertrauen in polnische Produkte seitens deutscher Unternehmer und reicht hin bis zu einem generellen Misstrauen gegenüber der polnischen Mentalität oder dem wenig ausgeprägten Kooperationswillen bei deutschen und polnischen Unternehmern. ${ }^{19}$

\section{Aktuelle Tendenzen grenzüberschreitender Unternehmenskooperationen}

Wenn die Grenzregionen an den neuen EU-Binnengrenzen sich in Zukunft noch stärker zu Motoren der europäischen Integration entwickeln sollen, dann müssen sie bereit sein, von den Grenzregionen in den alten Bundesländern bei aller Unterschiedlichkeit in den Voraussetzungen und Bedingungen zu lernen. Hinzu kommt die Notwendigkeit des Studiums von internationalen Erfahrungen mit ,cross-border cooperation' im weltweiten Kontext wie zum Beispiel an der US-amerikanisch-mexikanischen Grenze in Gestalt der ,Maquiladoras'-Industries, wo seit Mitte der 1960er Jahre erfolgreich zwischen mexikanischen und US-amerikanischen Firmen schon in der vierten Generation der ,Maquiladoras' kooperiert wird.

\section{Von der Lohnveredelung (,verlängerte Werkbank') zum Partner entlang der Wertschöpfungskette}

Während das Hauptmotiv für grenzüberschreitende Kooperationen für viele deutsche KMU lange vorrangig die Erschließung von Kostensenkungspotenzialen war, sind die polnischen Firmen immer mehr bestrebt, sich über Kooperationen in international verflochtene Wertschöpfungsketten deutscher oder anderer westlicher Unternehmen zu integrieren.

Es ist interessant zu beobachten, wie sich die Wandlungsprozesse im Inneren der polnischen Wirtschaft (starke Lohnerhöhungen) auf das Kooperationsverhalten der deutschen KMU ausgewirkt haben, denn nicht immer gelang es auf der polnischen Seite der Grenze, die gestiegenen Arbeitskosten durch eine höhere Produktivität auszugleichen.

Die in den 1990er Jahren stark anzutreffenden Lohnveredelungsgeschäfte verloren ihre bestimmende Rolle als Funktionsmuster für die grenzüberschreitenden Unternehmenskooperationen. ${ }^{20}$ Diese Form der ,verlängerten Werkbank' war zumeist mit einer Dominanz der deutschen Unternehmen verbunden, was sich nunmehr wandelt hin zu einer eher gleichberechtigten Kooperation entlang der gesamten Wertschöpfungskette. Auch polnische und tschechische Unternehmen in den Grenzregionen sind daran interessiert, komplexere und mit höherer Wertschöpfung verbundene Aufgaben zu übernehmen. Somit haben auch sektoral die Bekleidungs- und Möbelindustrie als die Domänen der Lohnveredelung in der $\mathrm{Zu}-$ sammenarbeit mit polnischen Firmen an Gewicht verloren. Mit den neuen Inhalten und Strukturen in den Kooperationsbeziehungen wird viel davon abhängen, wie es gelingt, In-

19 Vgl. Andrea Blaneck: Netzwerke und Kooperationen an der deutsch-polnischen Grenze, München 2005.

20 Lohnveredelungsgeschäfte sind eine Mischform von Unternehmenskooperation sowie Ex- und Importen, wobei in Ländern mit niedrigerem Lohnniveau Rohstoffe und Materialien bearbeitet werden beziehungsweise Komponenten, Bauteile und Baugruppen montiert werden (typische Branchen sind Bekleidung, Textilien, Möbel und Schuhe sowie die Autoindustrie). 
novationsprozesse, die die gesamte Wertschöpfungskette betreffen, arbeitsteilig und kooperativ zu bewältigen.

In den vergangenen Jahren haben internationale Technologieentwicklungen in den einzelnen Branchen, die Gegenstand der grenzüberschreitenden Unternehmenskooperationen sind, den Inhalt, Ablauf und vor allem die Anforderungen an die Kooperationen beeinflusst. Das hat auch den Zuschnitt der deutsch-polnischen und deutsch-tschechischen Unternehmenskooperationen verändert:

„Den polnischen Partnerunternehmen werden zunehmend ganzheitliche, mit höheren Qualifikationsanforderungen und höherer Wertschöpfung verbundene Aufgaben übertragen." ${ }^{21}$ Was die Zukunft anbetrifft, so wird sich der hier nur kurz skizzierte Strukturwandel in den Kooperationsinhalten noch stärker vollziehen, wenn es gelingt, die Unternehmen in den Grenzregionen in bestehende Clusterräume und unternehmerische Netzwerke einzubinden, die meist in den industriellen Metropolen angesiedelt sind (Poznan, Krakau und Dresden).

\section{Das ,twin location '-Konzept in der grenzüberschreitenden Kooperation ${ }^{22}$}

In dem Bemühen, auch weltweite Erfahrungen der Kooperation in Grenzregionen zu nutzen, stießen die verantwortlichen Manager der Wirtschaftsförderung in Brandenburg auf das bereits in der Praxis erprobte Konzept der ,Maquiladora'-Industrie an der US-amerikanischmexikanischen Grenze. ${ }^{23}$ Dabei galt und gilt es, auch die negative Seiten der ,Maquiladoras ‘ wie zum Beispiel zurückgehender Lebensstandard auf der mexikanischen Seite der Grenze oder Umweltschäden zu vermeiden. Interessant war und ist weiterhin die Nutzung von Investitions- und Lohnkostenunterschieden in Gestalt des ,twin location"-Modelles, um korrespondierende Gewerbegebiete beiderseits der Grenze zu entwickeln. Betriebswirtschaftlich ist dabei festzuhalten, dass Mischkalkulationen und Investitionen an einem Doppelstandort ein attraktives Investment verkörpern können. Das Modell erlaubt es, Schlussfolgerungen abzuleiten, wie die Fertigung auf die deutsch-polnische Seite der Grenzregion aufzuteilen ist beziehungsweise wie Entscheidungen zum Beispiel zur Gründung von Tochtergesellschaften in der benachbarten Wojewodschaft getroffen werden können.

Anwendung fand und findet dieses Konzept zum Beispiel in der Metallverarbeitung oder in Gestalt eines Twin-Call-Centers in Frankfurt Oder und Slubice. Bleibt für die Zukunft anzumerken: Das Modell eines Doppelstandortes an der deutsch-polnischen Grenze hat seine erste Bewährungsprobe bestanden und muss nun noch stärker an ausländische Investoren herangetragen werden, damit diese nicht mehr wie bisher die Grenzregionen, überspringen ‘ und nur in traditionellen Metropolen wie in Poznan, Lodz, Wroclaw, Warschau etc. operieren. So gesehen kann sich das Konzept von der Doppelstadt durchaus als ein Baustein auf dem Weg zur langfristigen Schaffung eines gemeinsamen deutsch-polnischen Wirtschaftsraumes entwickeln.

21 Ralph-Elmar Lungwitz: Jenseits der Lohnveredelung - Entwicklungstendenzen in der deutsch-polnischen Unternehmenskooperation, in: Harald Zschiedrich (Hrsg.): Wirtschaftliche Zusammenarbeit von Grenzregionen, Berlin 2011, S. 299-314.

22 Vgl. Irina Kania/Siegfried Behrendt: Das Konzept der Twin-Factory in der deutsch-polnischen grenzüberschreitenden Zusammenarbeit - Erfahrungen des Investor Center Ostbrandenburg, in: Harald Zschiedrich (Hrsg.): Wirtschaftliche Zusammenarbeit in Grenzregionen, Berlin 2011, S. 125-139.

23 Vgl. Regina Vellmer: Das Konzept der Maquiladoras - ein Muster für die deutsch-polnische grenzüberschreitende Unternehmenskooperation, in: Harald Zschiedrich (Hrsg.): Wirtschaftliche Zusammenarbeit in Grenzregionen, Berlin 2011, S. 213-226. 


\section{Schlussbemerkungen}

Die neuen Grenzregionen innerhalb der Europäischen Union können und müssen sich noch mehr profilieren als Motor und Labor von Internationalisierung und wirtschaftlicher Integration, wenn auch die Voraussetzungen dafür sehr unterschiedlich und nicht immer einfach sind. In diesem Prozess auf dem Wege zu gemeinsamen Wirtschaftsräumen sollten die Unternehmen und Unternehmer noch enger zusammenrücken. Sie müssen sich noch mehr interessieren und aktiv anbieten, um grenzüberschreitend Innovationsprozesse gemeinsam zu realisieren. In diesem Zusammenhang werden wechselseitiges Vertrauen und Handlungssicherheiten auf beiden Seiten zur entscheidenden Voraussetzung für das Zustandekommen wirtschaftlicher Kooperationen. Die Wirtschaftförderung muss noch mehr Anreize entwickeln, die direkt an die Interessen und Erfordernisse der Unternehmen anknüpfen.

Mit einem Blick in die Zukunft der deutsch-polnischen Kooperation - auch und gerade in den Grenzregionen - hat es Polens Botschafter Marek Prawda auf den Punkt gebracht: „Die deutsch-polnische Geschichte bleibt immer wichtig, aber zwischen den beiden Ländern/ Wirtschaften entsteht ein Raum, den man heute selbst gestalten kann. “24

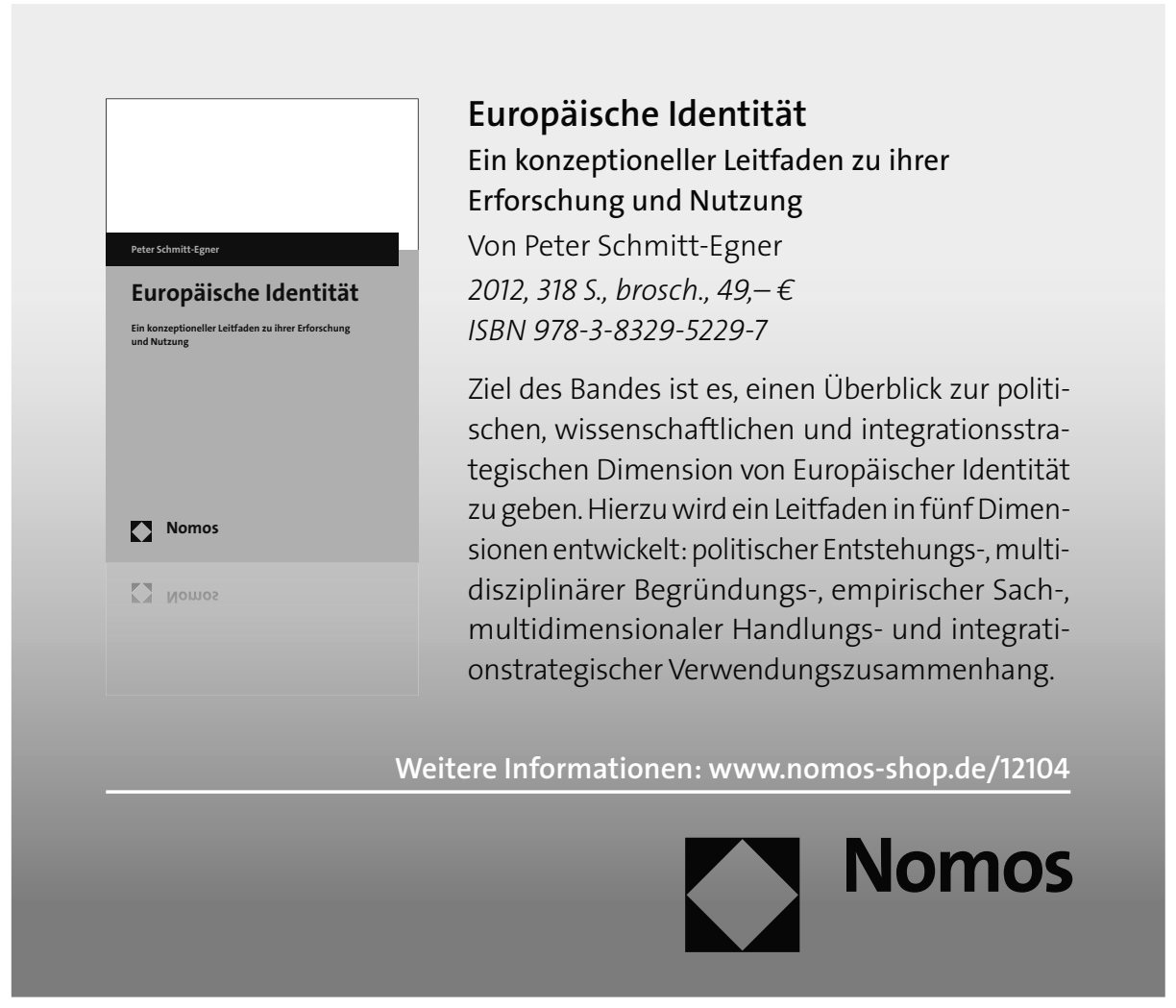

24 Vgl. Marek Prawda: Rede auf dem 57. Bundeskongress der Europa - Union Deutschland, 27. November, Berlin 2011. 